\title{
Schonfeld, I. S. and Chang, C.: Occupational Health Psychology. Springer Publishing Company, New York, 2017, 355 pp, \$50.20 (softbound)
}

\author{
Bruce A. Barron ${ }^{1}$ \\ Published online: 31 October 2018 \\ ๑) Springer Science+Business Media, LLC, part of Springer Nature 2018
}

The psychology of work is critical to employee mental and physical health. Occupational health psychology (OHP) is a complex subject because the mental health of employees not only impacts workplace performance and productivity; workplace stressors can adversely affect worker psychological and physical well-being. Therefore, understanding occupational health psychology is critically important regarding employee primary, secondary, and tertiary prevention of work-related injuries and illnesses. One of the major goals of Occupational Health Psychology is to provide the reader with relevant information that will further their knowledge in this interdisciplinary subfield of psychology.

Occupational Health Psychology is a comprehensive text that is logically organized into 11 chapters. The book starts with a brief history of OHP and relevant research methods, which is followed by chapters on the impact of psychosocial working conditions on mental health and the epidemiology of medical disease and OHP. While these chapters are well written and referenced, subsequent chapters provide a wealth of relevant information regarding workplace violence, organizational climate and leadership, occupational safety, work-life balance, and practical primary, secondary, and tertiary OHP interventions.

Overall, the text is expertly written by authoritative authors. Although published for undergraduate and graduate survey courses, the authors' assessment of depression, burnout, and other employee health conditions in addition to workplace factors such as organization and leadership should prove useful to most occupational health professionals, disability case managers, and others interested in maximizing employee well-being, performance, and productivity as well as minimizing work-related injury, illness, and disability. Readers should be aware that Occupational Health Psychology, while serving as an excellent reference tool for managing aggregate employee health policies and programs, would not be practical as a quick reference guide for timeconstrained practitioners in a busy clinic.
Bruce A. Barron

bruce_barron@urmc.rochester.edu

1 University of Rochester Medical Center, Rochester, USA 